\title{
Optocase Mini
}

\author{
Sanjay Sharma MD, \\ MSc (Epid), FRCS \\ Professor of Ophthalmology \\ \& Epidemiology, \\ Queen's University \\ Editor-in-Chief \\ optocase.com
}

\begin{abstract}
A 23 year old male presents with a lesion on his right upper lid. He says he first noticed the lesion approximately 6 weeks ago, at which time it was red and painful. Over the following weeks, the redness and pain subsided but he still has a visible nodule on the right upper lid. He reports no other complaints such as red eye, decreased vision, or diplopia. His past ocular and medical history is unremarkable.

On exam, a firm nontender nodule is palpated deep in the right upper lid close to the lid margin. The lesion does not appear inflamed. The patient also has evidence of some mild blepharitis, with a small amount of thick white discharge expressed from glands with gentle lid pressure.
\end{abstract}

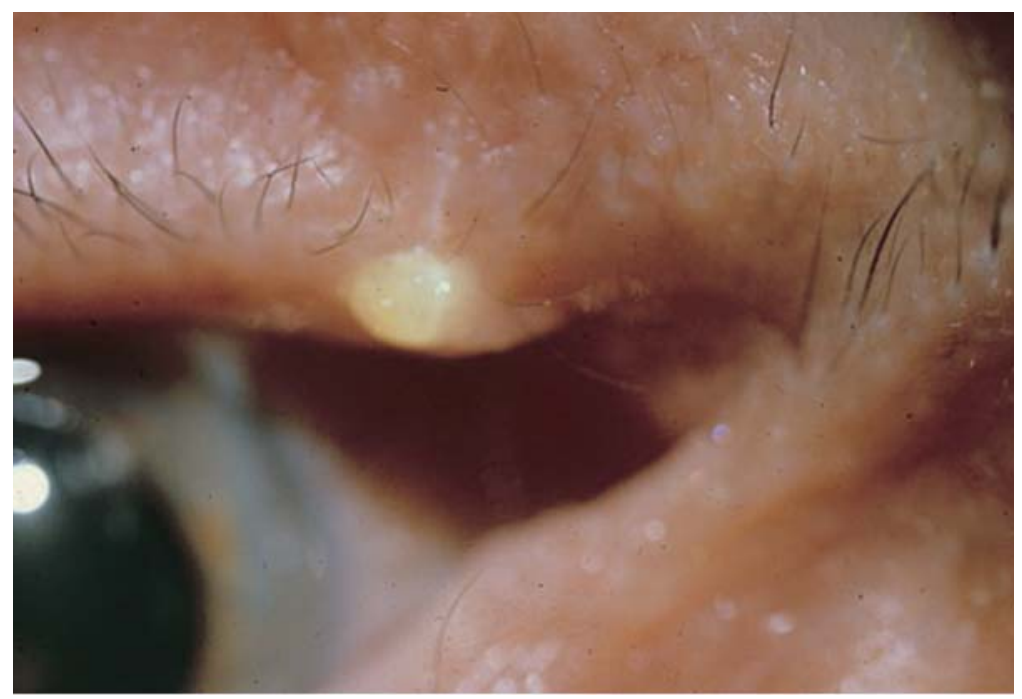

\section{Question: \\ What is the most likely diagnosis?}
(a) Stye
(b) Sebaceous cell carcinoma
(c) Chalazion
(d) Insect bite

\section{ANSWER TO OPTOCASE MINI}

\section{Answer: Chalazion}

A chalazion or meibomian cyst is a benign lesion of the eyelids occurring as a result of obstruction to a meibomian gland or gland of Zeis. They are most common in adults and occur equally in males and females. They are the most common inflammatory lesions of the eyelid.

\section{Presentation}

A chalazion is most often painless. It presents as a mild to moderately tender red swelling of the upper or lower eyelid. ${ }^{2}$ The onset of chalazion is typically over a few weeks, with the redness and tenderness subsiding as the lump remains. ${ }^{2}$ They are more common on the upper eyelids due to the increased number and length of meibomian glands on the upper eyelid.

Chalazia are most common solitary but can occasionally be in multiple locations. Upon palpation they are often felt deep within the lid, whereas a stye is more superficial and typically centred over an eyelash. Pres- 
sure exerted on lids occasionally produces a toothpaste-like discharge from meibomian glands (as opposed to the normally oily and clear discharge).

\section{Pathophysiology}

Approximately 30 meibomian glands line the posterior surface of the tarsus, functioning mainly to provide the lipid component of the tear film. Such lipids provide a hydrophobic barrier to prevent evaporation and tears spilling over the cheek.

Most chalazia occur as a result of obstruction to a meibomian gland. ${ }^{3}$ Following obstruction, the gland ruptures and releases its lipid products into surrounding eyelid soft tissue, triggering a lipogranulomatous reaction. ${ }^{4}$ Pathologically the inflammatory response consists of neutrophils, plasma cells, lymphocytes, histiocytes, and giant cells in a zonal configuration around a central lipid material. ${ }^{5}$ Early, inflammation appears as a suppurating granuloma, progressively evolving into a chronic mixed granulomatous inflammation and finally healing with the formation of granulation tissue and fibrosis. ${ }^{5}$ A chalazion is both benign and sterile, although occasionally secondary infection may occur. ${ }^{4}$

Occasionally, obstruction of zeisian sebaceous glands can also produce a chalazion, which can often be distinguished clinically by its more superficial location. ${ }^{5}$ A chalazion is distinct from a stye, which arises from an infected hair follicle on the lid margin and is characterized pathologically by an acute pyogenic inflammation with polymorphonuclear leukocytes and necrosis with pustule formation.

\section{Management and Recommendations}

A chalazion is most often a clinical diagnosis made relatively easily with a consistent history and exam. However, multiple benign, pre-malignant, as well as malignant conditions can mimic a chalazion. ${ }^{1}$

Apart from biopsy (which is not routine for a typical appearing lesion), no other diagnostic tests are used in identifying chalazia. Bacterial cultures are usually negative, although occasionally species such as Staphylococcus aureus, Staphylococcus albus, or Propionibacterium acnes may be isolated. Recently, research has been conducted into the possibility of using infrared photography to image and identify pathology in meibomian glands of the eyelid, although this technology has not yet been using in a clinical setting.

Acute chalazia are generally treated conservatively at first, as many resolve with or even without such measures (spontaneously resolution generally takes many months though). This involves warm wet compresses which help promote dilation and drainage of duct contents. Patients should be instructed to perform twice daily warm compresses (3-5 minutes) and massage with either fingers or cotton tips. ${ }^{2}$ The massage should be directed over the lesion in the direction of the eyelashes, helping to promote the release of gland contents. Conservative treatment has a success rate of approximately $46 \% .^{6}$ Incision and drainage is also necessary for persistent lesions. ${ }^{6}$

\section{Follow-Up}

All chalazia should be followed-up by an Optometrist or Ophthalmologist until resolution is noted. Failure to respond to treatment should raise suspicion of an alternative diagnosis, especially sebaceous cell carcinoma.

\section{CONTINUING EDUCATION FOR OPTOMETRISTS}

Are you short on CE credits? Optocase.com is the easiest way to earn COPE-approved credits online on your own schedule. Our credits are accepted by all Canadian provinces, and we always offer at least one free case on the site.

For more information, visit optocase.com. $\bullet$

\section{REFERENCES}

1. Ozdal PC, Codere F, Callejo S, Caissie AL, Burnier MN. Accuracy of the clinical diagnosis of chalazion. Eye (Lond). Feb 2004;18(2):135-138.

2. Gilchrist H, Lee G. Management of chalazia in general practice. Aust Fam Physician. May 2009;38(5):311-314.

3. Carter SR. Eyelid disorders: diagnosis and management. Am Fam Physician. Jun 1998;57(11):2695-2702.

4. Duarte AF, Moreira E, Nogueira A, Santos P, Azevedo F. Chalazion surgery: advantages of a subconjunctival approach. J Cosmet Laser Ther. Sep 2009;11(3):154-156.
Dhaliwal U, Arora VK, Singh N, Bhatia A. Cytopathology of chalazia. Diagn Cytopathol. Aug 2004;31(2):118-122.

6. Goawalla A, Lee V. A prospective randomized treatment study comparing three treatment options for chalazia: triamcinolone acetonide injections, incision and curettage and treatment with hot compresses. Clin Experiment Ophthalmol. Nov 2007;35(8):706-712. 